\title{
Novel SACS Mutation Deviates from the French Canadian ARSACS Phenotype
}

\author{
E.D. McKenzie, P.N. Sharma, J.S. Parboosingh, Forge Canada Consortium*, O. Suchowersky
}

Can J Neurol Sci. 2014; 41: 88-89

Autosomal recessive spastic ataxia of Charlevoix-Saguenay (ARSACS) is a genetic condition caused by mutation in the spastic ataxia of Charlevoix-Saguenay (SACS) gene, which clinically manifests as a slowly-progressive ataxic gait disorder. Other typical characteristics include spasticity, dysarthria, distal muscle wasting, foot deformities, truncal ataxia, absence of sensory-evoked potentials in lower limbs, and myelinated retinal fibres. ${ }^{1-3}$ The disorder was first characterized in a population in north-eastern Quebec, where two founder mutations were identified. A diversity of SACS mutations have since been described in Quebec, all with the classical French Canadian ARSACS phenotype., ${ }^{2,3}$ This condition appears to be one of the more common causes of autosomal recessive cerebellar ataxia. More than 70 ARSACS-associated mutations have been reported worldwide, with significant variations in phenotype described..$^{3,4}$ It appears to be one of the more common causes of autosomal recessive cerebellar ataxia. We present a case of ARSACS due to a novel mutation in the SACS gene which deviates from the French Canadian phenotype.

\section{Case Report}

Our patient is a 35 year old right-handed woman, who has been followed for progressive cerebellar ataxia in the Movement Disorders Clinic since 2005. The patient was born at 42 weeks following a normal pregnancy; developmental milestones were normal. However, her family observed a clumsy gait since infancy, with uncoordinated arm movements. Cognitively, the patient developed normally. Gait and balance peaked at approximately ten years-of-age and have since deteriorated. However, she reports that fine motor skills have improved with practice over time. The patient has mild non-progressive dysarthria and intermittent dysphagia (especially for lowviscosity beverages). She is also aware of bladder urgency and frequency. She had a fasciotomy for pes cavus and release surgery for tendon contractures in adolescence.

The patient is of British and German ethnic descent. Her parents are first cousins; neither parent has any neurological abnormalities. The patient has an older brother who suffers from attention deficit hyperactivity disorder. The family indicated that a distant cousin on the father's side may be affected by a condition similar to that of the patient.

On cranial nerve examination, the patient exhibits saccadic smooth pursuit, horizontal and vertical gaze-evoked nystagmus, oscillopsia, and dysarthria with scanning speech. Fundi are normal. An intention tremor is present in both arms and legs, and she has wide-based ataxic gait. Sensory testing in the arms is normal; she has decreased vibration sense to the knees bilaterally. Testing of strength reveals mild weakness and wasting in her hands, and muscle wasting and weakness below the knee. The patient demonstrates brisk tendon reflexes and bilateral extensor plantar responses. She shows dystonic inversion of the feet, pes cavus, and hammer toes (See video).

\section{Investigations}

Biochemical tests, including vitamin $\mathrm{B}_{12}$, vitamin $\mathrm{E}$, folate, CK, albumin, alphafetoprotein and apolipoprotein, were unremarkable. Electrophysiological testing revealed slowing of nerve conduction velocities, with decreased distal latencies, lowered CMAPs and absent SNAPs. Bone densitometry showed osteoporosis. Brain magnetic resonance imaging (MRI) showed atrophy of the cerebellar vermis. Genetic analysis of DNA extracted from whole blood for Friedreich's ataxia, autosomal dominant spinocerebellar ataxia 1,2,3,6,7, and 8, and the two common French Canadian mutations of ARSACS, SACS (NM_014363.4) c.8844delT and c.7504C $>$ T (originally published as $6594 \mathrm{delT}$ and $5254 \mathrm{C}>\mathrm{T}$ ) yielded negative results.

Following informed consent, whole blood samples for DNA extraction were obtained from the patient, her parents and brother. These were submitted to the Finding Of Rare Disease Genes in Canada (FORGE) project, an initiative which uses next-generation techniques to sequence the exomes of patients to identify disease-causing genetic changes. Exome sequencing resulted in the identification of sixteen variants for which the patient was homozygous, whereas her parents and brother were heterozygous at these loci. Of these sixteen homozygous variants, the mutation causative of her condition was determined to be a frameshift insertion in the SACS gene, c.9284dupC, confirmed via Sanger sequencing.

\footnotetext{
From the Department of Medicine (Neurology) (EDM, OS) Departments of Medical Genetics, Pediatrics, and Psychiatry (OS), University of Alberta, Edmonton;

Department of Medical Genetics and Alberta Children's Hospital Research Institute for Child and Maternal Health (PNS, JSP), University of Calgary, Calgary, Alberta,

Canada. *Forge Canada Consortium members listed in Acknowledgements section. Received May 29, 2013. Final Revisions Submitted July 22, 2013. Correspondence to: Oksana Suchowersky, Medical Genetics, Pediatrics, and Psychiatry, Department of Medicine, University of Alberta, 233.32 Walter Mackenzie Health Science Centre, 8440 - 112 Street, Edmonton, Alberta, T6G 2B7, Canada. Email: Oksana.Suchowersky@albertahealthservices.ca.
} 


\section{Discussion}

Our patient presented with a slowly progressive cerebellar ataxia with upper and lower motor neuron signs. The consanguineous background suggested the condition was autosomal recessive in nature. Over a dozen forms of autosomal recessive conditions have been described in which cerebellar ataxia is a prominent sign. ${ }^{1}$ Genetic diagnosis is essential for distinguishing among the cerebellar ataxias due to phenotypic and genotypic overlap.

Over 70 disease-causing mutations have been identified in SACS.$^{3,4}$ Nearly all of the identified mutations are spread out over the last exon, which at approximately $13 \mathrm{~kb}$ is one of the largest exons in the human genome. ${ }^{3}$ Only the two French Canadian founder mutations are routinely tested with targeted analysis in many provinces of Canada. ${ }^{3}$ Not unexpectedly, our patient was normal for the French Canadian SACS mutations.

Our patient was found to have a novel frameshift mutation in SACS predicted to cause a premature stop codon and a truncated protein (p.Ala3096Cysfs*2) resulting in the absence of the last third of the protein. Sacsin, the protein encoded by the SACS gene, is highly expressed in Purkinje cells and thought to interact with the Hsp70 chaperone machinery as part of the cell response to aggregation-prone mutant proteins associated with neurodegenerative disease. ${ }^{3}$

Our patient's ARSACS-causing mutation was discovered using whole exome sequencing, which involves sequencing all the protein-coding regions of the genome (the exome). Although the exome is only approximately $1 \%$ of the genome, it is estimated to contain $85 \%$ of mutations causing disease-related traits..$^{5}$ This technique allows rare or novel mutations to be detected without the prohibitive cost of whole-genome sequencing, and is advantageous for the detection of mutations in individuals with genetically heterogeneous conditions, eliminating the gene-by-gene approach. ${ }^{5}$

The clinical presentation of our patient varies from the French Canadian ARSACS phenotype. Our patient does not have hypermyelinated retinal fibers, the distinguishing feature of ARSACS in the French Canadian population.,3 A case of ARSACS without hypermyelinated retinal fibers in a consanguineous Japanese family has been previously described. ${ }^{4}$ Additionally, dysarthria in the French Canadian population is typically progressive, whereas our patient's dysarthria is nonprogressive and mild. .,3 $^{2}$

Clinically, no individual feature definitively distinguishes ARSACS from the many other autosomal recessive cerebellar ataxias, necessitating genetic testing to confirm a suspected case. ${ }^{1-4}$ Furthermore, the growing diversity of reported mutations in the SACS gene indicates that many disease-causing mutations will be undetected by the currently available targeted mutation analysis. Exome sequencing is recommended for patients for whom ARSACS is a consideration. While treatment for autosomal recessive spinocerebellar ataxias is currently limited to management of symptoms and disability, genetic confirmation of the type of cerebellar ataxia is necessary for appropriate genetic counselling with respect to prognosis, risk to relatives, and risk to future pregnancies. The findings reported here illustrate the importance of integrating next-generation genetic technologies such as exome sequencing into clinical diagnostics.

\section{ACKNOWLEDGEMENTS}

The authors thank the patient and her family for their cooperation in this study.

FORGE Canada Consortium*: Finding of Rare Disease Genes in Canada; Steering Committee: Kym Boycott (leader; University of Ottawa), Jan Friedman (co-lead; University of British Columbia), Jacques Michaud (co-lead; Université de Montréal), Francois Bernier (University of Calgary), Michael Brudno (University of Toronto), Bridget Fernandez (Memorial University), Bartha Knoppers (McGill University), Mark Samuels (Université de Montréal), Steve Scherer (University of Toronto).

\section{FUNDING ACKNOWLEDGEMENT}

This work was funded by the Government of Canada through Genome Canada, the Canadian Institutes of Health Research and the Ontario Genomics Institute (OGI-049). Additional funding was provided by Genome Quebec and Genome British Columbia.

Dr. Suchowersky is funded by the Toupin Research Foundation at the University of Alberta.

\section{REFERENCES}

1. Anheim M, Tranchant C, Koenig M. The autosomal recessive cerebellar ataxias. N. Engl. J. Med. 2012;366(7):636-46.

2. Thiffault I, Dicaire MJ, Tetreault M, Huang KN. Diversity of ARSACS Mutations in French-Canadians. Can J Neurol Sci. 2013:40:60-6.

3. Bouhlal Y, Amouri R, Euch-Fayeche El G, Hentati F. Autosomal recessive spastic ataxia of Charlevoix-Saguenay: an overview. Parkinsonism Relat Disord. 2011;17(6):418-22.

4. Miyatake S, Miyake N, Doi H, et al. A novel SACS mutation in an atypical case with autosomal recessive spastic ataxia of Charlevoix-Saguenay (ARSACS). Intern Med. 2012;51:2221-6.

5. Choi M, Scholl UI, Ji W, et al. Genetic diagnosis by whole exome capture and massively parallel DNA sequencing. Proc Natl Acad Sci USA. 2009;106(45):19096-101. 